\title{
ARTICLE
}

\section{Gamma-ray transmission evaluation using imaging plate designed for $\mathrm{X}$-ray radiography}

\author{
Hiroyuki Miyamaru $^{\mathrm{a}}$, Isao Murata ${ }^{\mathrm{b}}$, Ryoichi Taniguchi ${ }^{\mathrm{a}}$, Hiromoto Shimomura ${ }^{\mathrm{a}}$ and Shuichi Okuda ${ }^{\mathrm{a}}$ \\ ${ }^{a}$ Radiation Research Center, Osaka Prefecture University, Gakuen-cho 1-2, Nakaku, Sakai, Osaka 599-8531, Japan; \\ ${ }^{b}$ Graduate School of Engineering, Osaka University, Yamada-oka 2-1, Suita, Osaka 565-0871, Japan
}

\begin{abstract}
A commercial imaging plate (IP) designed for X-rays and a cesium-137 radiation exposure device were employed to evaluate gamma-ray transmission over the wide surface area of a specimen. A thin copper sheet was used as an intensifying material to enhance the sensitivity of the IP to gamma rays. The response of the copper-covered IP to the transmitted gamma ray was expressed as a two-dimensional gray-tone digital image. The relationship was experimentally determined between the transmission of copper plates with different thickness and corresponding gray values on the digital image. The gray value of the specimen was compared with the one of the copper plate exposed simultaneously on the same image to evaluate gamma-ray transmission. A two-dimensional array of stainless steel balls was fabricated as an example specimen with non-uniform thickness and many voids and its transmission was evaluated as a test. The transmission evaluated from the image agreed well with the one calculated using PHITS and the one measured with an ion chamber. The image of a thick concrete brick showed that a difference in gray values appeared between the center and edge parts. Spread of Compton scattering gamma rays was found to affect the image of a thick specimen.
\end{abstract}

Keywords: gamma-ray transmission; imaging plate; gray-tone image; gamma-ray shielding; cesium radiation source

\section{Introduction}

Because of the Fukushima nuclear power plant disaster, attention has been given to gamma-ray shielding materials to safely handle rubble containing radioactive cesium. Composite materials such as concrete containing grains of heavy metal have been proposed as a replacement or substitute for lead because of the problems with handling and cost. Furthermore, radiation-protective clothing containing powder and a mesh wire of high-density materials such as tungsten and lead has been developed for reducing the exposure of a human body to radiation. Gamma-ray transmission is often used for estimation of radiation shielding property. A parallel beam of monoenergetic gamma rays is attenuated with a relation, which is given by the exponential expression,

$$
I=I_{o} \exp \left(-\mu_{m} t\right)
$$

where $I_{o}$ and $I$ are the incident and transmitted gamma-ray intensities, $t$ is the thickness of an absorbing medium as measured in $\mathrm{g} / \mathrm{cm}^{2}$ and $\mu_{m}$ is the mass attenuation coefficient. The ratio of $I / I_{o}$ represents quantitative value of radiation attenuation. In general, the gamma-ray transmission is measured with a

*Corresponding author. Email: miyamaru@riast.osakafu-u.ac.jp radiation detector and a narrow beam from a radiation source. This method, however, does not seem suitable for non-uniform and complex specimens because the transmission may be different depending on the local position of the specimen. Moreover, only the averaged value is evaluated by this conventional method. Thus, a more practical method is required for evaluating gamma-ray transmission for composite and non-uniform specimens. In this study, a commercial imaging plate (IP) designed for X-ray was covered with a thin copper sheet to intensify gamma-ray response. A specimen was set on the copper-covered IP and was exposed with cesium gamma rays to obtain a two-dimensional gray-tone digital image. In this paper, we discuss the possibility of the gamma-ray transmission evaluation from the obtained digital image.

\section{Experimental setup}

The experiment was performed in the exposure room of the Radiation Research Center of Osaka Prefecture University. Figure $\mathbf{1}$ presents the schematic for gamma-ray exposure. An industrial radiography exposure device containing a $1.8 \mathrm{Ci}$ cesium-137 radioactive source was used for gamma-ray exposure. The device consisted of a lead container, source guide tube, and remote control conduit. The gamma-ray 
source was introduced to the head of the guide tube via a manual crank-out mechanism. A source position corresponding to the exposure working position was set about $1 \mathrm{~m}$ above ground level. A measurement specimen was set $80 \mathrm{~cm}$ below the source position. The CR ST-VI imaging plate (Fuji), which is a commercial product for X-ray radiography [1,2], was used for the experiment. The IP covered with a 0.3-mm-thick copper sheet was set below the specimen. The role of the copper sheet is described later. The ion chamber (VICTOREEN 550-3-T) was set below the IP with a lead aperture $30 \mathrm{~mm}$ in diameter to measure the gamma-ray transmission of a local area of the specimen. After gamma-ray exposure, the IP was brought to a readout unit (FUJI AC-7/ST) and processed to form a gray-tone computed radiography (CR) image. The dimensions of the IP were $20 \mathrm{~cm} \times 25 \mathrm{~cm}$, and one pixel was $0.1 \mathrm{~mm}^{2}$. Output images were subsequently processed using the Image $1.45 \mathrm{~s}$ software for evaluating the gamma-ray transmission. All images were processed using a neighborhood mean filter (4 pixels) for smoothing and were converted into 8-bit gray-tone images. The dose for exposure was low so that no afterimage was left behind on the IP. The IP was used repeatedly after the readout procedure.

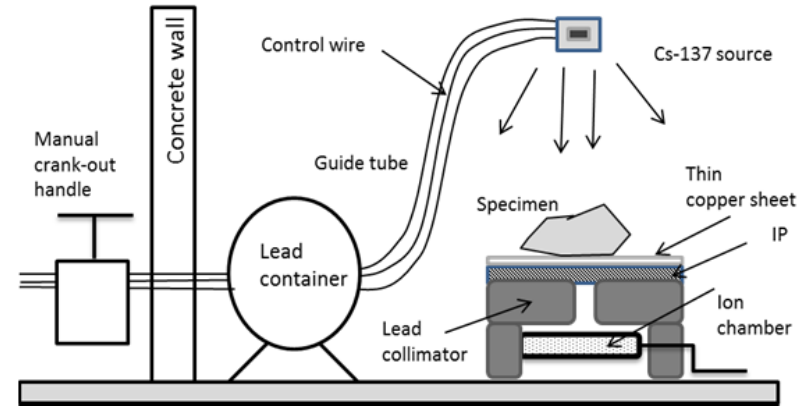

Figure 1. Experimental setup for gamma-ray transmission measurement using IP.

\section{Experimentals}

\subsection{Gamma-ray response of IP}

Figure 2 shows the response of the IP exposed to a dose of $15 \mu \mathrm{Gy}$ as a gray-tone digital image. The right half of the image corresponds to the part of the IP covered with a 0.3-mm-thick copper sheet before exposure, and the left half shows the bare IP surface. Images of the lead plates with different thicknesses are shown as three rectangles. The gray value at each pixel corresponds to the intensity of the radiation response. The left part displayed in light gray corresponds to the area that did not respond to the gamma rays; in contrast, the deep dark color at the covered part indicates a more intense response. Since the copper sheet was thin, the exposure intensity to cesium gamma rays at each part was expected to be almost the same value. Nevertheless, covering the IP with a copper sheet produced a higher response against gamma rays. Our method of using a thin metal sheet on the IP for increasing the sensitivity to high-energy gamma rays is similar to that of studies using a metal plate/phosphor screen [3,4]. As with previous studies, the thin copper sheet possibly acts as an intensifying material that converts a gamma ray into $\mathrm{X}$-rays and electrons.

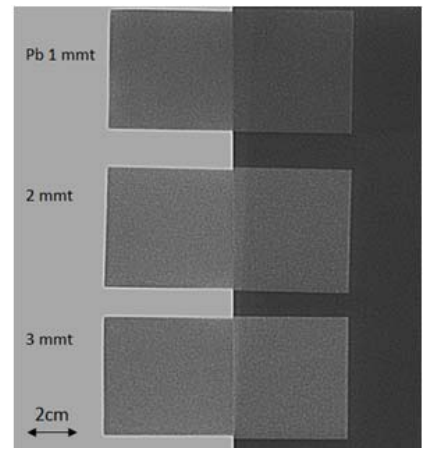

Figure 2. Gray-tone digital image of the IP exposed to cesium gamma rays. Right side is covered with a thin copper sheet during exposure. The images of lead plates with different thicknesses are shown as three rectangles on the boundary.

The response of the generated X-rays and electrons was recorded on the IP; consequently, the response of the IP covered with the copper sheet shows much higher sensitivity than that of bare IP. The images of the lead plates on the copper-covered side were compared; the lead plate with $1 \mathrm{~mm}$ thick shows deep gray and the one with 3mm thick is rather bright. This result suggests that the IP shows different gray color in accordance with the transmission characteristic of a gamma-ray exposed specimen. The relation between gamma-ray transmission and the response of the copper-covered IP is examined experimentally in a following subchapter. Fading effect of the image was not observed in our study. This transmission image using the copper-covered IP and gamma-ray exposure is completely reproducible. To intensify the sensitivity, the $0.3 \mathrm{~mm}$-thick copper sheet was always placed in front of the IP throughout our study.

\subsection{Relationship between gray value and gamma-ray transmission}

Figure 3 shows the gray-tone image of copper plates with $2-8 \mathrm{~mm}$ thickness. The difference in thickness is clearly expressed by the relative differences in the gray-tone color of a single image. In order to clarify the relationship between the gray value and the thickness of copper, the representative gray value of each copper plate was determined through the following procedure. A region-of-interest (ROI) that was $2 \mathrm{~cm}^{2}$ was selected from the image of the plate, and a histogram was calculated from the distribution of gray values in the selected ROI. The mean gray value of the histogram was finally taken as the representative gray value of the copper plate for a specific thickness. For dense materials like the copper plate, the histogram showed a 
symmetrical shape, as shown in the figure; however, specimens that include voids show complicated shapes as demonstrated later.

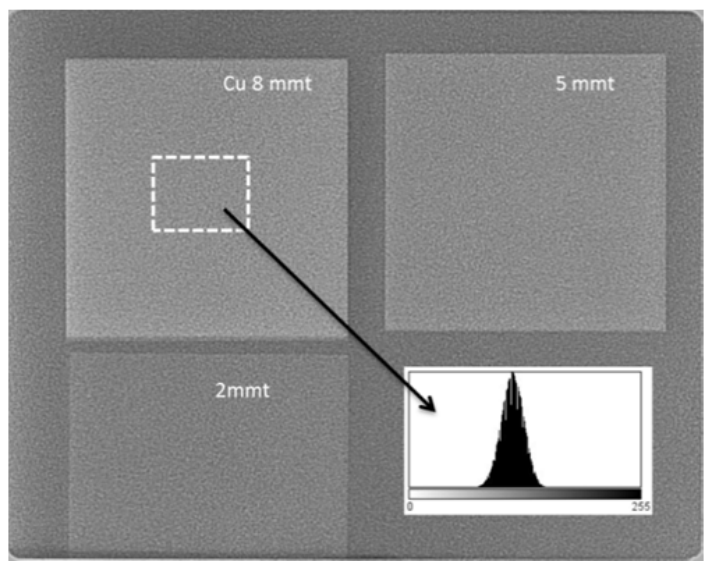

Figure 3. Gray-tone digital image of copper plates with different thicknesses. The histogram at right bottom represents the distribution of the gray values in the dashed area.

The gray value employed here is not an absolute value but is relative. Although the gray value can be adjusted using software as a digital image, relative relation of the gray value at each position in one image can be maintained. In every exposure, several copper plates with known thickness were simultaneously exposed with a target specimen. The gray values of copper plates were used as reference to evaluate the gamma ray transmission in each specimen position of a single image. Mean gray values of the IP are plotted versus exposure dose in Figure 4. These values were taken in the exposure condition using the copper-covered IP. These values were evaluated from the IP area without a specimen. The response of the IP used here showed linear dependency with gamma-ray dose in the range we employed for exposure.

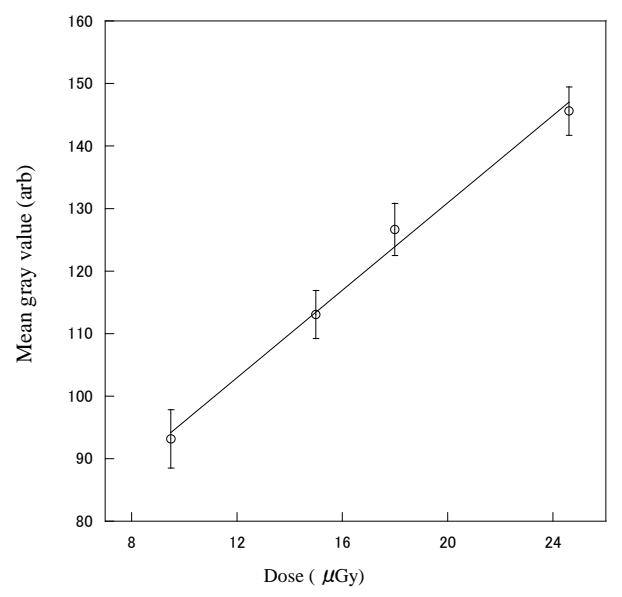

Figure 4. Relationship between mean gray value and gamma-ray dose for exposure. The uncertainty is standard deviation of the gray value in the histogram.
Figure 5 shows the relationship between thickness of copper and mean gray values taken in our study. The uncertainty was the standard deviation of the gray value in the histogram. The relationship shows a linear dependency. The transmission of cesium gamma ray for individual thickness is calculated with above denoted equation and mass attenuation coefficient of the XCOM data [5]. From the calculation result, the copper thickness of 2-15 $\mathrm{mm}$ corresponds to the range from 0.88 to 0.38 of transmission. Experimental result clearly indicates that the evaluated mean gray value has linear dependency with the gamma-ray transmission.

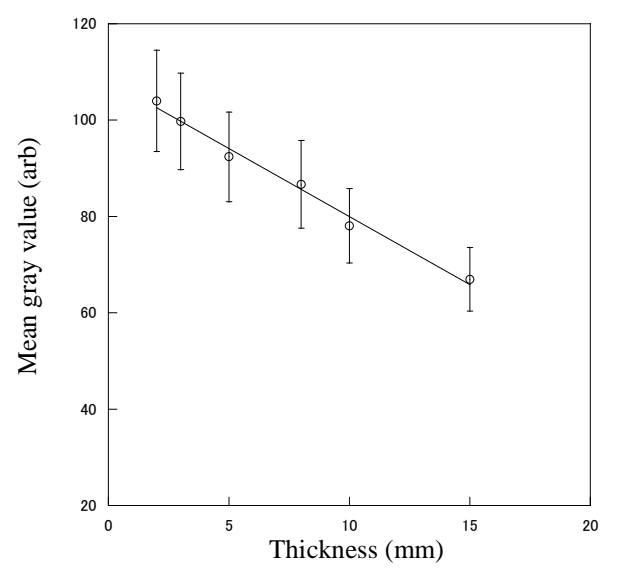

Figure 5. Relationship between mean gray value and thickness of copper. The uncertainty is standard deviation of the gray value in the histogram.

\subsection{Two-dimensional stainless steel ball array}

A two-dimensional array of stainless steel balls was fabricated as an example specimen with non-uniform thickness and many voids, and its gamma-ray transmission was evaluated from the image. Figure 6(a) shows the gray-tone image of the ball array, and Figure 6(b) shows the three-dimensional image of the selected region. The gray scale of 3-D image is adjusted manually for appearance. Stainless steel balls with1.27 $\mathrm{cm}$ diameters were aligned two-dimensionally in a plastic cage, and two copper plates that were 3- and 7mm-thick were positioned besides the array for comparison. In the two-dimensional image, the shape of the array can be clearly identified, but the distribution of the gray value inside a single ball is unclear. The gap between the balls corresponds to the voids and is expressed with a deep gray color. The histogram in Figure 6(a) was calculated from the gray values in the dotted circle of the image. The dotted circle was selected as a ROI, and the gamma-ray transmission at this region was evaluated. The obtained histogram does not show a symmetrical single peak like the copper plate above but shows two peaks: the primary peak at the light gray-tone side is due to the balls, and the secondary one is due to the voids. 


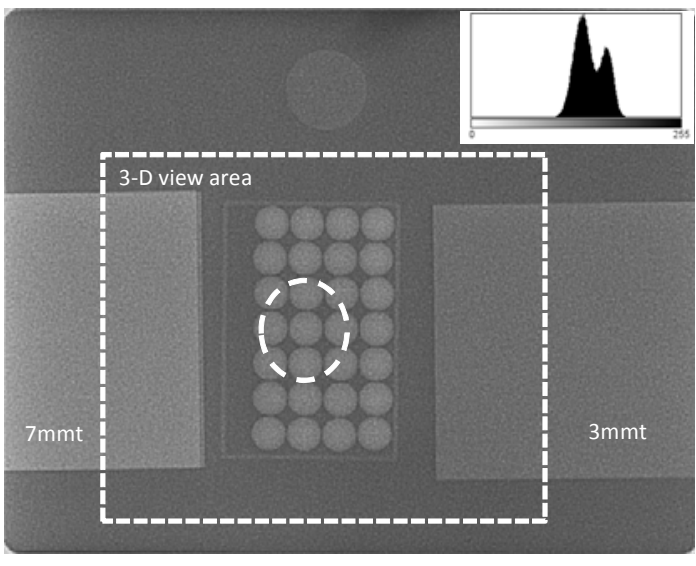

Figure 6. (a) Gray-tone digital image of stainless steel ball array. The gamma-ray transmission at the dashed circle area was evaluated. The histogram in the figure represents the distribution of the gray values of the target area.

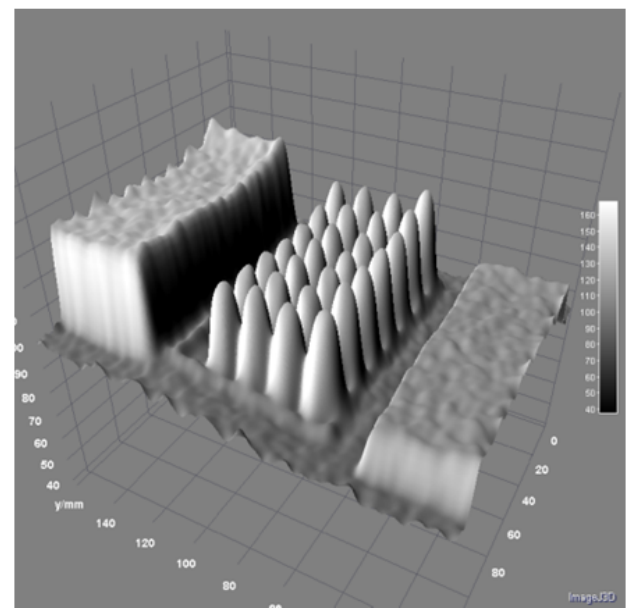

Figure 6. (b) Three-dimensional gray-tone image of the ball array. Corresponding area is the dashed rectangle in Fig. 6(a).

In order to evaluate the gamma-ray transmission at the ROI, the integrated value of the gray values over the histogram was employed as a representative value instead of the averaged gray value in this case. The ROI with the same size was specified for the individual copper plate, and the integrated value was calculated in the same manner. The integrated gray value of the ball array was compared with those of the copper plates and corresponding thickness of copper was estimated. The gamma-ray transmission of the ball array was finally evaluated from the calculation with corresponding thickness. The transmission at the ROI was experimentally measured using the ion chamber. A Monte Carlo simulation of the gamma-ray transmission was performed using PHITS [6] under the calculation conditions including the same structure of the ball array. Table 1 summarizes the results of the transmission of the ball array. The uncertainty of the transmission evaluated from the IP was evaluated from the standard deviation of the integrated gray value. The uncertainty in the calculation is the deviation of the tallies calculated with different conditions in which the gamma-ray beam positions against the ball array are slightly changed. Although the uncertainty range is somewhat wide, the transmission evaluated from the image agreed well with both the calculated and measured results.

Table 1. Evaluated transmission value of the ball array.

\begin{tabular}{ccc}
\hline IP & Ion chamber & Calculation \\
\hline $70 \pm 12 \%$ & $69 \pm 4 \%$ & $70.0 \pm 1.5 \%$ \\
\hline
\end{tabular}

\subsection{Image of concrete brick}

The gray-tone image of a flat concrete brick was obtained as an example of a large and thick specimen. The brick was homogeneous, and its thickness was also uniform. The dimensions of the brick were $20 \mathrm{~cm} \times 10$ $\mathrm{cm} \times 4 \mathrm{~cm}$, and the density was $2.2 \mathrm{~g} / \mathrm{cm}^{3}$. Figure 7 shows the gray-tone and three-dimensional images of the concrete brick. The change in the gray value can be
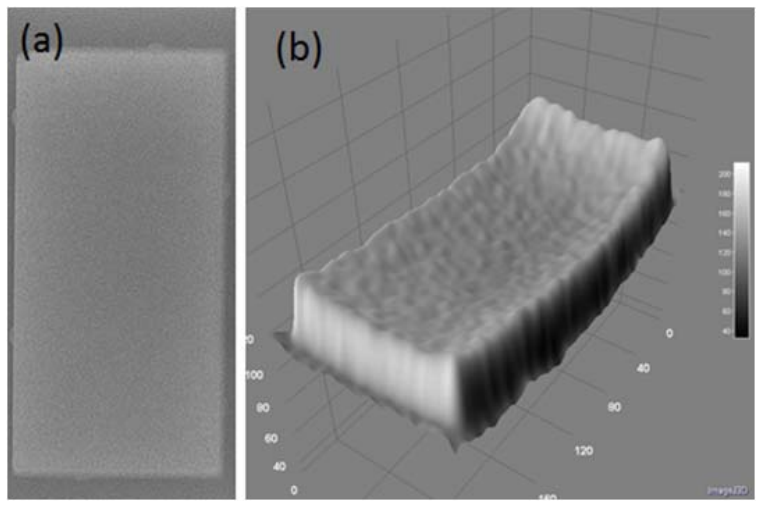

Figure 7. (a) Gray-tone digital image of the concrete brick. (b) Three-dimensional image.

observed; the color of the center part is a deeper gray rather than that of the edge. The gamma-ray transmission as measured with the ion chamber was about $56 \%$, and this was the same for the entire surface area of the brick. In the image, the gray value of the edge part corresponded to the correct value, but the value at the center part indicated a large increase in transmission. The obtained image shows disagreement with the gamma-ray transmission property of the brick. Presence of deep gray at the center part can be explained due to Compton scattering gamma rays generated in the brick. Angular spread of the scattering gamma ray exposes the pixel locating far from the initial interaction position. Consequently, the overlap of this exposure becomes remarkable at the center part. We found that the influence of scattering gamma rays should be taken into account in evaluating the transmission correctly for a thick specimen. 


\section{Conclusion}

In our study, we employed a commercial X-ray imaging plate covered with a thin copper plate to establish a practical method for evaluating the cesium gamma-ray transmission of various materials. The transmission of the ball array as evaluated from the image agreed well with both the experimental and simulation results. The gray-tone image of the thick concrete brick showed different gray values at the edge and center parts, resulting in the influence of scattering gamma rays. Our evaluation method is currently not suitable for a thick specimen. Appropriate correction of the image for a thick specimen is currently under investigation. Further study is in progress to establish a practical evaluation method for gamma-ray transmission using an imaging plate.

\section{References}

[1] M. Sonoda, M. Takano, J. Miyahara and H. Kato, Computed radiography utilizing scanning laser stimulated luminescence, Radiology 148 (1983), pp. 833-838.
[2] K. Takahashi, Progress in science and technology on photostimulable $\mathrm{BaFX}: \mathrm{Eu}^{2+}(\mathrm{X}=\mathrm{Cl}, \mathrm{Br}, \mathrm{I})$ and imaging plates, J. Luminescence, 100 1-4 (2002), pp. 307-315.

[3] D. S. O'Keeffe and R. W. McLeod, Computed radiography as a gamma ray detector-dose response and applications, Physics in Medicine and Biology 49 (16) (2004), pp. 3559-3572.

[4] S. Taniguchi, A. Yamadera, T. Nakamura and A. Fukumura, Measurement of radiation tracks for particle and energy identification by using imaging plate, Nucl. Instr. and Meth. in Phys. Res. A 413 (1998), pp. 119-126.

[5] L. Gerward, N. Guilbert, K. B. Jensen and H. Levring, X-ray absorption in matter, Reengineering XCOM, Radiation Physics and Chemistory 60 (1-2) (2001), pp. 23-24.

[6] K. Niita, N. Matsuda, Y. Iwamoto, H. Iwase, T. Sato, H. Nakashima, Y. Sakamoto and L. Sihver, PHITS: Particle and Heavy Ion Transport Code System, Version 2.23, JAEA-Data/Code 2010-022 (2010). 